\title{
Surface and bulk modes for periodic structures of negative index materials
}

\author{
Ruey-Lin Chern, ${ }^{1,2, *}$ Chien C. Chang, ${ }^{2,1, \dagger}$ and C. Chung Chang ${ }^{2}$ \\ ${ }^{1}$ Institute of Applied Mechanics, National Taiwan University, Taipei 106, Taiwan, Republic of China \\ ${ }^{2}$ Division of Mechanics, Research Center for Applied Sciences, Academia Sinica, Taipei 115, Taiwan, Republic of China
}

(Received 15 June 2006; revised manuscript received 10 August 2006; published 3 October 2006)

\begin{abstract}
In this paper, we investigate surface and bulk modes for periodic structures made of negative index materials in one and two dimensions. The negative index material is a composite medium consisting of a network of thin wires and a periodic array of split ring resonators. In different ranges of frequencies, we identify five types of modes: surface plasmon (SP) modes for TE polarization, magnetic surface plasmon (MSP) modes for TM polarization, trapped modes or resonant cavity modes for both TM and TE polarizations, asymmetric surface (AS) modes for TE polarization, and some bulk modes for both polarizations in the range of negative material properties. In particular, we will discuss band flattening and broadening of SP and MSP modes, explain the trapped modes in terms of large positive dielectric constants, examine the properties of AS modes by an interface condition, and use the Rayleigh quotient to account for possibly infinite degeneracy of SP and MSP modes in two dimensions. All of the physical properties are computed by an interfacial operator approach in which we introduce an interfacial variable to measure the local strengths of various surface modes.
\end{abstract}

DOI: $10.1103 /$ PhysRevB.74.155101

PACS number(s): 42.70.Qs, 78.20.Bh, 02.70.-c, 02.70.Bf

\section{INTRODUCTION}

Materials with both negative electric permittivity and magnetic permeability have been proposed some 30 years ago by Veselago. ${ }^{1}$ Unusual electromagnetic properties are expected due to the left handedness between the $\mathbf{E}, \mathbf{H}$ fields and the wave vector $\mathbf{k}$. Accordingly, the index of refraction $n$ would be negative for these materials. However, there are no natural materials with negative permeability. Until recently, double negative materials $(\varepsilon<0, \mu<0)$ in an effective sense can be realized by Pendry et al. using a network of thin wires, ${ }^{2}$ combined with a periodic array of split ring resonators. ${ }^{3}$ This composite medium could be considered homogeneous at wavelengths much larger than the size and spacing of the elements of the medium, and has an effective electric permittivity of the form

$$
\varepsilon_{n}(\omega)=1-\frac{\omega_{p}^{2}}{\omega^{2}},
$$

where $\omega_{p}$ is the effective plasma frequency, and an effective magnetic permeability of the form

$$
\mu_{n}(\omega)=1-\frac{F \omega^{2}}{\omega^{2}-\omega_{0}^{2}},
$$

where $\omega_{0}$ is the resonant frequency of the split ring and $F$ is the fractional area of the unit cell occupied by the interior of the split ring. The composite medium exhibits simultaneously negative permittivity and permeability in the microwave regime, with $\omega_{p}$ and $\omega_{0}$ in the $\mathrm{GHz}$ range. ${ }^{4}$ Recently, negative permeability has been realized in the $100 \mathrm{THz}$ range. ${ }^{5}$ It must be noted that realistic double negative metamaterials must be dispersive, ${ }^{4}$ otherwise the causality constraint ${ }^{6}$ will be violated. This is consistent with the permittivity model (1) and permeability model (2) of the composite medium described above.

Because of its special format (2), the magnetic permeability bears some similarity to the electric permittivity, but has one particular point that is not shared by the latter. Figure 1 shows the frequency dependence of the effective permittivity (1) and permeability (2). On the one hand, both have negative values in a range of frequencies: the electric permittivity is negative in $\left[0, \omega_{p}\right]$, while the magnetic permeability is negative in $\left[\omega_{0}, \omega_{m}\right]$, where $\omega_{m}=\omega_{0} / \sqrt{1-F}$. Besides, both $\varepsilon_{n}$ and $\mu_{n}$ have a zero crossing at the upper limit of the range: the electric permittivity at $\omega_{p}$ and the magnetic permeability at $\omega_{m}$. On the other hand, the magnetic permeability turns from positive to negative infinity in crossing the resonance frequency $\omega_{0}$. The present study is aimed to study the band structures of periodic structures made of negative index materials, and in particular, we will investigate the consequences of the interplay between the electric permittivity and the magnetic permeability in different ranges of frequencies.

In the simplest case of a planar interface between the negative index material and a material with positive con-

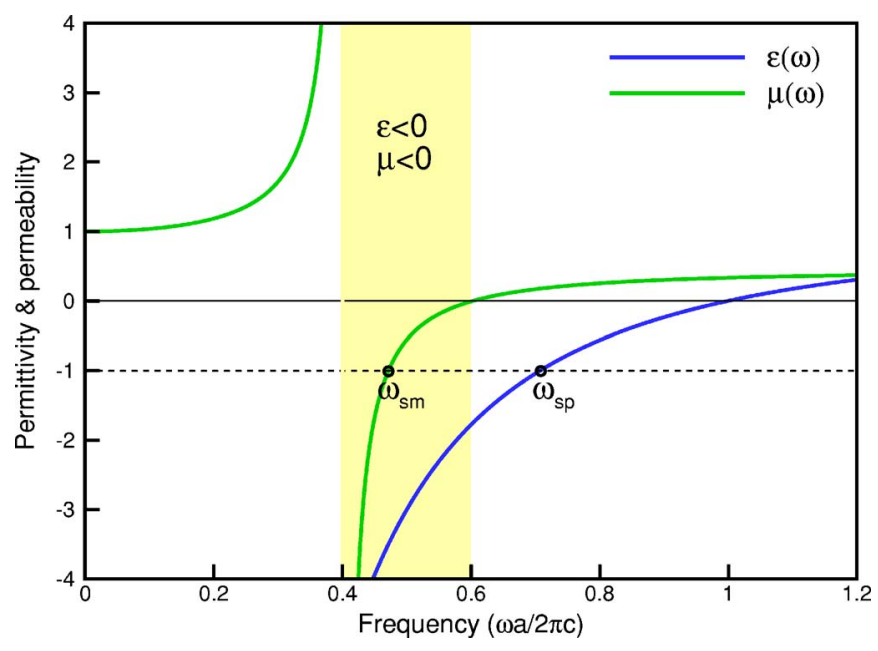

FIG. 1. (Color online) The effective $\varepsilon$ and $\mu$ of the negative index material for $\omega_{p} a / 2 \pi c=1.0, \omega_{0} a / 2 \pi c=0.4$, and $F=0.56$. The yellow region corresponds to the frequency range where the medium has both negative $\varepsilon$ and $\mu$. The black circles denote the locations of $\omega_{\mathrm{sp}}$ and $\omega_{\mathrm{sm}}$ for $\varepsilon_{d}=1$ and $\mu_{d}=1$. 
stants $\epsilon_{d}$ and $\mu_{d}$, there are two types of surface modes living in the neighborhood of the interface. One is called the surface plasmon (SP) mode, and its frequency (at large off-line wave numbers) is determined by $\epsilon_{d}+\epsilon_{n}(\omega)=0$, which yields the surface plasma frequency

$$
\omega_{\mathrm{sp}}=\frac{\omega_{p}}{\sqrt{1+\varepsilon_{d}}} .
$$

The SP is a result of the coupling of photons with electrons. The other is a magnetic analog of the surface plasmon, called the magnetic surface plasmon (MSP), and its frequency (at large off-line wave numbers) is determined by $\mu_{d}+\mu_{n}(\omega)$ $=0$, which yields the magnetic surface plasma frequency

$$
\omega_{\mathrm{sm}}=\sqrt{\frac{\mu_{\infty} \omega_{m}^{2}+\mu_{d} \omega_{0}^{2}}{\mu_{\infty}+\mu_{d}}} .
$$

In the case of an array of split ring resonators placed in a magnetic field, the induced currents flowing in the cylinders produce magnetization (magnetic polarization). It appears as if magnetic monopoles were flowing on opposite ends of the cylinders. ${ }^{7}$ On a scale much larger than the size of split ring resonators, the above phenomenon behaves like a magnetic plasma. Under suitable conditions, coupling of magnetic plasma with electromagnetic fields may give rise to MSP. It is noted that MSP have similar features of surface magnons (SM) which can be found in ferromagnetic and antiferromagnetic materials. ${ }^{8-10}$ However, MSP and SM have different physical origins, for SM waves come from the coupling of photons with electron spins, and tend to die out above $\mathrm{GHz}$ frequencies. ${ }^{5}$

It has been difficult to compute eigenmodes and band structures of frequency-dependent materials. The major difficulty is due to a nonlinear formulation of the eigenvalue problem. ${ }^{11}$ Another difficulty comes from the highly localized nature of the solutions, which is more evident for SP (or MSP) modes. Several approaches have been proposed to study the frequency-dependent problem, including plane wave expansion method, ${ }^{12}$ transfer matrix method, ${ }^{13}$ multiple scattering theory or Korringa-Kohn-Rostoker (KKR) method, ${ }^{14-16}$ layer-KKR method, ${ }^{17}$ finite-difference timedomain method ${ }^{18}$ and multiple multipole method. ${ }^{19}$ Recently, vectorial eigenmode expansion method ${ }^{20}$ and cutting surface method $^{11}$ were also applied to this type of problem. In this study, we will compute the various types of modes for onedimensional as well as two-dimensional periodic structures made of negative index materials, and gives the detailed physical and mathematical account of the observed mode patterns. These modes will be computed in the framework of the interfacial operator approach, recently developed by the present authors..$^{21,22}$

It is of general interest to see what happens to SP and MSP modes if the interface is not planar, or the interface is not a single plane, but is such complicated as in the periodic structures. Indeed, we will show that the patterns of the modes for periodic structures made of the negative index material with optical properties (1) and (2) can be divided into five types (a) SP modes distributed around $\omega_{\text {sp }}$, (b) MSP modes distributed around $\omega_{\mathrm{sm}}$, (c) trapped modes or reso- nance cavity (RC) modes lying immediately above the frequency $\omega_{0}$, (d) asymmetric surface (AS) modes lying immediately below $\omega_{0}$, and (e) other bulk modes in the range of negative material properties. Then, we will discuss band flattening and broadening of SP and MSP modes, explain the trapped modes in terms of large positive dielectric constants, examine the properties of AS modes by an interface condition, and use the Rayleigh quotient to account for possibly infinite degeneracy of SP and MSP modes in two dimensions.

\section{INTERFACIAL OPERATOR APPROACH}

The time-harmonic electromagnetic wave equations for linear and isotropic materials in two dimensions are given by

$$
\begin{aligned}
& -\frac{1}{\varepsilon} \nabla \cdot\left(\frac{1}{\mu} \nabla E\right)=\left(\frac{\omega}{c}\right)^{2} E, \\
& -\frac{1}{\mu} \nabla \cdot\left(\frac{1}{\varepsilon} \nabla H\right)=\left(\frac{\omega}{c}\right)^{2} H
\end{aligned}
$$

for TM and TE modes, respectively. For periodic structures, it is sufficient to solve the underlying problem on one unit cell along with Bloch's condition as the boundary condition

$$
\begin{aligned}
& E\left(\mathbf{r}+\mathbf{a}_{i}\right)=e^{i \mathbf{k} \cdot \mathbf{a}_{i}} E(\mathbf{r}), \\
& H\left(\mathbf{r}+\mathbf{a}_{i}\right)=e^{i \mathbf{k} \cdot \mathbf{a}_{i}} H(\mathbf{r}),
\end{aligned}
$$

where $\mathbf{k}$ is the wave vector and $\mathbf{a}_{i}((i=1,2)$ are the lattice translation vectors. The resultant eigensystem for Eqs. (5) and (6) can be written as

$$
\mathcal{L}(\Lambda) \phi=\Lambda \phi,
$$

where $\Lambda=\omega^{2} / c^{2}$ is the eigenvalue and $\phi$ is the eigenfunction which can be either the $E$ or $H$ field. For frequencydependent materials, the eigensystem (9) no longer has a standard format since the eigenvalue itself appears in the differential operator. If Eq. (9) is solved by discretizing it in a straightforward manner, for example, by a finite-difference scheme, we will obtain a nonlinear eigensystem

$$
\mathbf{A}(\Lambda) \mathbf{x}=\Lambda \mathbf{x},
$$

where $\mathbf{A}$ is the matrix system, and $\mathbf{x}$ is the eigenvector. This is one type of nonlinear eigenvalue problem, that is, nonlinear in eigenfrequency. However, if both the permittivity and permeability of the material have analytical forms, we are able to reformulate the original nonlinear eigenvalue problem as a standard one.

In our previous studies, ${ }^{21,22}$ we have proposed the interfacial operator approach to compute surface plasmon modes for periodic structures made of dispersive metals as well as polar materials. In this paper, we extend this approach to be applicable to negative index materials with the effective permittivity model (1) and permeability model (2).

The basic idea is first to deal with the eigensystems (5) and (6) in the strict insides of the dielectric and the negative index material separately, so that $\varepsilon$ and $\mu$ can be moved out 
of the operator in either region. In the strict inside of the dielectric,

$$
\begin{aligned}
& -\nabla^{2} E=\varepsilon_{d} \mu_{d} \Lambda E, \\
& -\nabla^{2} H=\varepsilon_{d} \mu_{d} \Lambda H,
\end{aligned}
$$

where $\varepsilon_{d}$ and $\mu_{d}$ are the permittivity and permeability, respectively, of the surrounding dielectric material. In the strict inside of the negative index material,

$$
\begin{aligned}
& -\nabla^{2} E=\mu_{\infty}\left(\Lambda-\Lambda_{p}\right)\left(\frac{\Lambda-\Lambda_{m}}{\Lambda-\Lambda_{0}}\right) E, \\
& -\nabla^{2} H=\mu_{\infty}\left(\Lambda-\Lambda_{p}\right)\left(\frac{\Lambda-\Lambda_{m}}{\Lambda-\Lambda_{0}}\right) H,
\end{aligned}
$$

where $\Lambda_{p}=\omega_{p}^{2} / c^{2}, \Lambda_{0}=\omega_{0}^{2} / c^{2}$, and $\Lambda_{m}=\omega_{m}^{2} / c^{2}$. Here, $\mu_{\infty}=1$ $-F$ is the high frequency limit of the effective permeability, and $\omega_{m}=\omega_{0} / \sqrt{1-F}$ is the effective magnetic plasma frequency. Equations (13) and (14) can be further rearranged as

$$
\begin{aligned}
\Lambda^{2} E-\Lambda L_{1} E+L_{2} E & =0, \\
\Lambda^{2} H-\Lambda L_{1} H+L_{2} H & =0,
\end{aligned}
$$

where

$$
L_{1}=\Lambda_{p}+\Lambda_{m}-\frac{1}{\mu_{\infty}} \nabla^{2}, \quad L_{2}=\Lambda_{p} \Lambda_{m}-\frac{\Lambda_{0}}{\mu_{\infty}} \nabla^{2} .
$$

Equations (11), (13), (12), and (14) are connected, respectively, by the interface conditions

$$
\left(\frac{1}{\mu} \frac{\partial E}{\partial n}\right)_{S}=0, \quad\left(\frac{1}{\varepsilon} \frac{\partial H}{\partial n}\right)_{S}=0,
$$

where $\partial / \partial n$ denotes the derivative in the surface normal direction, and $[\cdots]_{S}$ the jump across the interface $S$ between the dielectric and the negative index material. The interface conditions (18) are obtained by integrating both sides of the eigensystem (5) and (6) over a thin box located on the interface, and taking the limit as the box height goes to zero. Applying the permittivity and permeability models (1) and (2), the interface conditions (18) are rearranged as

$$
\begin{gathered}
\left.\frac{\Lambda_{m}}{\mu_{d}} \frac{\partial E}{\partial n}\right|_{+}-\left.\frac{\Lambda_{0}}{\mu_{\infty}} \frac{\partial E}{\partial n}\right|_{-}=\Lambda S_{E}, \\
\left.\frac{\Lambda_{p}}{\varepsilon_{d}} \frac{\partial H}{\partial n}\right|_{+}=\Lambda S_{H}
\end{gathered}
$$

so that the eigenvalue $\Lambda$ appears only on the right-hand side, where

$$
\begin{gathered}
\left.S_{E} \equiv \frac{1}{\mu_{d}} \frac{\partial E}{\partial n}\right|_{+}-\left.\frac{1}{\mu_{\infty}} \frac{\partial E}{\partial n}\right|_{-}, \\
\left.S_{H} \equiv \frac{1}{\varepsilon_{d}} \frac{\partial H}{\partial n}\right|_{+}-\left.\frac{\partial H}{\partial n}\right|_{-}
\end{gathered}
$$

are the weighted differences of the normal derivatives of the $E$ and $H$ fields, respectively, across the interface, with + and
- denoting the dielectric and the negative index material sides, respectively.

Apparently, Eqs. (11) and (15), supplemented by Eq. (19), cannot be formulated as a standard eigensystem in terms of the $E$ field, for the right-hand sides of Eqs. (19) and (20) contain the derivatives of $E$ or $H$ field. Likewise, Eqs. (12), (16), and (20) cannot be formulated as a standard eigensystem for the $H$ field either. Nevertheless, this difficulty can be removed by employing the interfacial operator approach in finite difference formulation. ${ }^{21,22}$ With this approach, the eigensystem (10) can be recast into the following form:

$$
\left(\Lambda^{2}-\Lambda \mathbf{B}-\mathbf{C}\right) \mathbf{y}=0,
$$

and thus further reduced to

$$
\left[\begin{array}{ll}
\mathbf{0} & \mathbf{I} \\
\mathbf{C} & \mathbf{B}
\end{array}\right]\left[\begin{array}{c}
\mathbf{y} \\
\mathbf{y}^{\prime}
\end{array}\right]=\Lambda\left[\begin{array}{c}
\mathbf{y} \\
\mathbf{y}^{\prime}
\end{array}\right]
$$

where $\mathbf{y}^{\prime}=\Lambda \mathbf{y}, \mathbf{B}$ and $\mathbf{C}$ are constant matrices. The eigensystem (24) can be solved by standard eigenvalue solvers, and has the same eigenvalue $\Lambda$ as the original eigensystem (10), although the new eigenvector $\mathbf{y}$ is slightly different from the original eigenvector $\mathbf{x}$. However, they can be converted back and forth between each other.

\section{RESULTS AND DISCUSSION}

The primary interest of the present study is to understand the interplay between the effective electric permittivity $\epsilon$ and magnetic permeability $\mu$. The interplay consists mainly of the product $\epsilon \mu$, as suggested by Eqs. (11) and (12) for the dielectric, Eqs. (13) and (14) for the negative index material, and the interface conditions between them, dictated in Eqs. (19) and (20). In the present study, we use the following parameters for the permittivity (1) and permeability (2): $\omega_{p} / 2 \pi=10^{10} 1 / \mathrm{s}, \omega_{0} / 2 \pi=4 \times 10^{9} 1 / \mathrm{s}$, and $F=0.56$, which are in the same range as in Ref. 4. In nondimensional units, we choose $\omega_{p} a / 2 \pi c=1$ and $\omega_{0} a / 2 \pi c=0.4$ for the lattice constant $a=3 \mathrm{~cm}$. It follows that $\omega_{m} a / 2 \pi c=0.603$ and $\mu_{\infty}$ $=1-F=0.44$.

\section{A. One-dimensional structures}

Surface plasmon modes. SP modes have been studied for a semi-infinite metal and a metal slab. ${ }^{23}$ For periodic structures, SP modes have also been investigated for photonic crystals made of dispersive metals. ${ }^{18,19,21,24}$ On the other hand, MSP modes were reported for a left-handed material of a semi-infinite plane and a slab. ${ }^{25,26}$ In this study, we present MSP modes for periodic structures made of negative index materials. Figure 2 shows the dispersion relation at the zone edge $(k a / 2 \pi=0.5)$ for one-dimensional layered structures with thickness ratio $t / a=0.2$. It is shown that in addition to two branches of SP modes for TE polarization with frequencies approaching the surface plasma frequency $\omega_{\text {sp }}[\mathrm{cf}$. Eq. (3)] at large off-line wave number $\beta$ (component of the wave number parallel to the interface), there are two branches of MSP modes for TM polarization with frequencies approaching the magnetic surface plasma frequency $\omega_{\text {sm }}$ [cf. Eq. (4)]. It is noted that the asymptotic frequencies of SP or MSP 


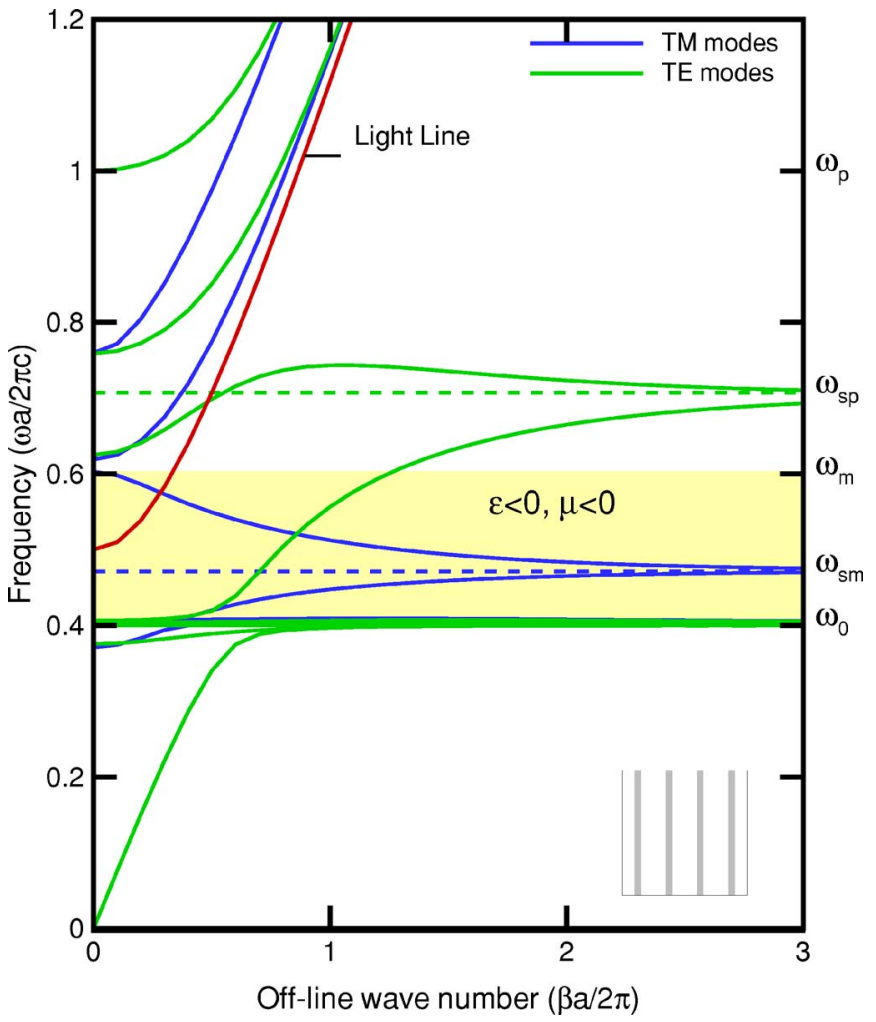

FIG. 2. (Color online) The dispersion relations at $k a / 2 \pi=0.5$ for a one-dimensional layered structure of negative index material of thickness $t / a=0.2$ where $\omega_{p} a / 2 \pi c=1.0, \omega_{0} a / 2 \pi c=0.4$, and $F$ $=0.56$.

modes for periodic structures is the same as for a semiinfinite plane and a slab. ${ }^{23,25,26}$ For $\varepsilon_{d}=1$ and $\mu_{d}=1$, we have $\omega_{\mathrm{sp}} a / 2 \pi c=0.7071$ and $\omega_{\mathrm{sm}} a / 2 \pi c=0.4714$, the locations of which are denoted by black circles in Fig. 1. Note also that $\omega_{\text {sm }}$ is located in the range $\omega_{0}<\omega<\omega_{m}$ where both $\varepsilon_{n}$ and $\mu_{n}$ are negative, and the composite medium is left handed. Besides, the higher band of MSP modes has a negative group velocity over the whole range of $\beta$. Figure 3 shows the dispersion relation for the thickness ratio $t / a=0.8$. It is shown that for a larger fraction of the negative index material, the frequency of the higher band of SP modes begins with the bulk plasma frequency $\omega_{p}$ at $\beta=0$ and approaches $\omega_{\mathrm{sp}}$ at large $\beta$, with a relatively large value of negative group velocity. Both Figs. 2 and 3 show that all the modes to the right of the light line will become surface modes (approaching $\omega_{\mathrm{sp}}$ or $\omega_{\mathrm{sm}}$ ) or RC modes (approaching $\omega_{0}$ from above) at the large limit of $\beta$. It is therefore expected that for large off-line wave number, propagation of the electromagnetic waves is prohibited except for the frequencies near three resonant frequencies $\omega_{0}, \omega_{\mathrm{sp}}$, and $\omega_{\mathrm{sm}}$.

Asymmetric surface modes. In addition to the two pairs of SP and MSP modes, Fig. 2 also shows two branches of asymmetric surface (AS) modes for TE polarization with frequencies approaching the resonant frequency $\omega_{0}$ from below. This is in contrast to the branches of SP or MSP modes with frequencies approaching $\omega_{\mathrm{sp}}$ or $\omega_{\mathrm{sm}}$ from both above and below. Figure 4 shows two AS modes at $\beta a / 2 \pi=1$ and two AS modes at $\beta a / 2 \pi=2$ for TE polarization at the zone cen-

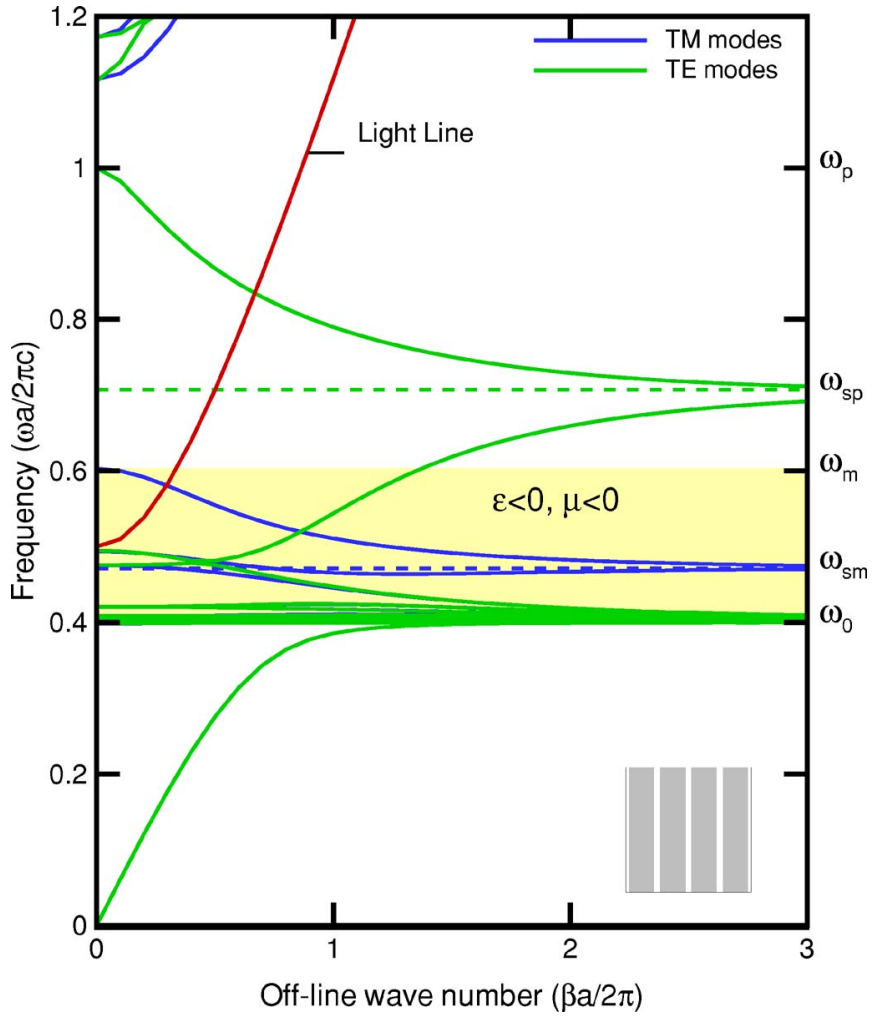

FIG. 3. (Color online) The dispersion relations at $k a / 2 \pi=0.5$ for a one-dimensional layered structure of negative index material of thickness $t / a=0.8$ where $\omega_{p} a / 2 \pi c=1.0, \omega_{0} a / 2 \pi c=0.4$, and $F$ $=0.56$.

ter $(k=0)$. The eigenfrequencies of the two AS modes approach the asymptotic frequency $\omega_{0}$ much faster than for SP or MSP modes. This type of surface modes appear only for TE polarization, which is consistent with the observation for the cases of a semi-infinite plane ${ }^{25}$ and a slab. ${ }^{26}$ Note also that the field decays much more rapidly inside the negative index material than outside. This can be understood from the interface condition at $\omega=\omega_{0}$,

$$
\left.\frac{\partial H}{\partial n}\right|_{-}=-\left.\left(\frac{\omega_{p}^{2}-\omega_{0}^{2}}{\varepsilon_{d} \omega_{0}^{2}}\right) \frac{\partial H}{\partial n}\right|_{+} .
$$

In the present case, the slope of the $H$ field (at the interface) on the side of the negative index material is $5.25\left[\left(\omega_{p}^{2}\right.\right.$ $\left.\left.-\omega_{0}^{2}\right) / \varepsilon_{d} \omega_{0}^{2}=5.25\right]$ times that on the dielectric side. This is the reason why these surface modes are said to be asymmetric. Existence of AS modes can be further explained through the dispersion relation of surface modes for TE polarization in a more general form ${ }^{27}$

$$
\beta=\frac{\omega}{c}\left(\frac{\varepsilon_{d} \varepsilon_{n}\left(\varepsilon_{d} \mu_{n}-\varepsilon_{n} \mu_{d}\right)}{\varepsilon_{d}^{2}-\varepsilon_{n}^{2}}\right)^{1 / 2} .
$$

Substituting $\varepsilon_{n}$ and $\mu_{n}$ with Eqs. (1) and (2) for the negative index material and taking the limit $\beta \rightarrow \infty$, we obtain 

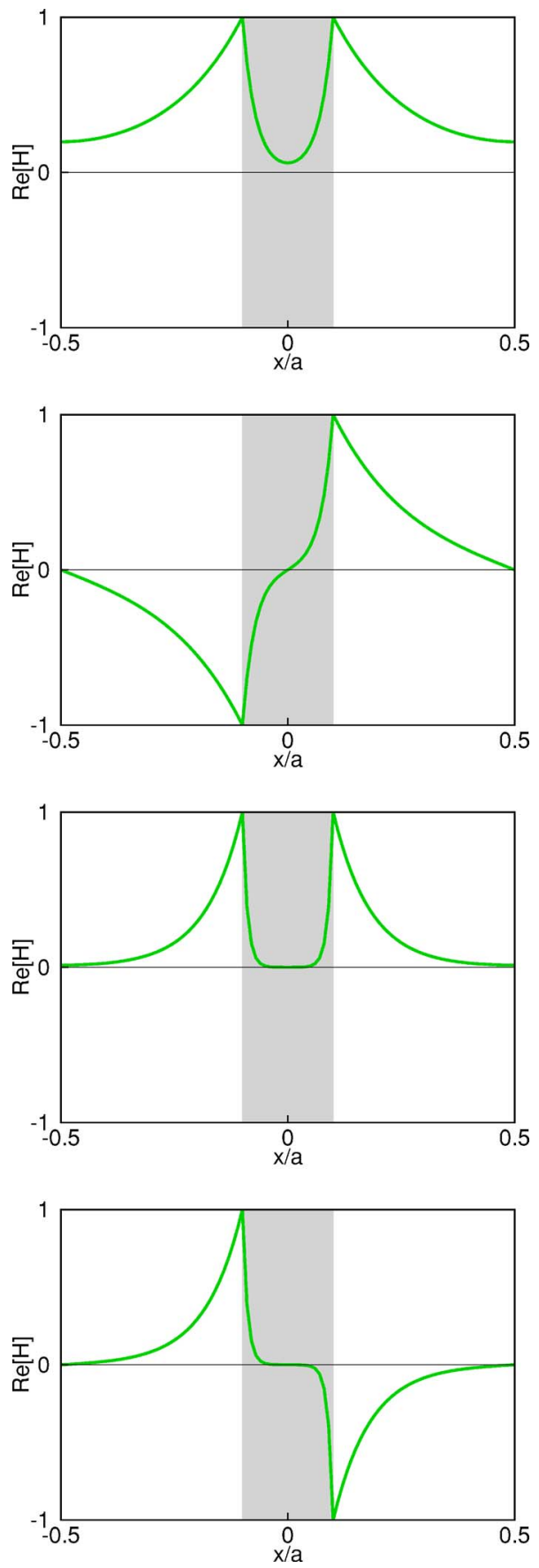

FIG. 4. (Color online) Two AS modes with $\omega a / 2 \pi c=0.3968$ and 0.3971 at the off-line wave number $\beta a / 2 \pi=1$ (upper figures), and two AS modes with $\omega a / 2 \pi c=0.3996$ at $\beta a / 2 \pi=2$ (lower figures) for TE polarization at the zone center $(k=0)$ for a onedimensional layered structure of negative index material of thickness $t / a=0.2$ where $\omega_{p} a / 2 \pi c=1.0, \omega_{0} a / 2 \pi c=0.4$, and $F=0.56$.

$$
\begin{aligned}
\left(\varepsilon_{d}^{2}-1\right) \omega^{6}+ & {\left[2 \omega_{p}^{2}-\left(\varepsilon_{d}^{2}-1\right) \omega_{0}^{2}\right] \omega^{4}-\omega_{p}^{2}\left(\omega_{p}^{2}+2 \omega_{0}^{2}\right) \omega^{2} } \\
+ & \omega_{p}^{4} \omega_{0}^{2}=0 .
\end{aligned}
$$

The above equation can be solved to yield the asymptotical solutions $\omega \rightarrow \omega_{\mathrm{sp}}=\omega_{p} / \sqrt{1+\varepsilon_{d}}$ as well as $\omega \rightarrow \omega_{0}$.
Resonant cavity modes. Refer again to Figs. 2 and 3. In a small range lying immediately above the resonant frequency $\omega_{0}, \varepsilon_{n} \mu_{n}$ becomes very large and the negative index material behaves like a cavity filled with a medium of very large refractive index. Most of the fields are trapped in the negative index material, and a large number of branches of bulk modes, called resonant cavity (RC) modes, are intensively gathering around the resonant frequency $\omega_{0}$ from above for both TM and TE polarizations. For a large fraction of the negative index material, there are even more RC modes with most of the fields being concentrated inside the medium. Figure 5 shows four typical RC modes for TM polarization with $\omega a / 2 \pi c=0.4$ at the zone center $(k=0)$ and $\beta=0$ for the thickness ratio $t / a=0.4$. These mode structures have strong oscillatory patterns in the negative index material and nearly null fields outside. A similar feature is also observed for TE polarization. In fact, these modes resemble the RC modes in photonic crystals made of polar materials. ${ }^{20}$

\section{B. Two-dimensional structures}

Infinite degeneracy. First of all, we are concerned with the grid resolution. Figure 6 shows the distribution of eigenfrequencies for TE modes for a square array of square cylinders of negative index material with half-width $w / a=0.2$. It is shown that the frequencies are little dependent on the grid level except in two distinct regions: one below the resonant frequency $\omega_{0}$ (RC modes) and one around the surface plasma frequency $\omega_{\mathrm{sp}}$ (SP modes). As expected, the modes intensively distributed above $\omega_{0}$ are the RC modes. However, contrary to the one-dimensional structures that have only two branches of SP modes, the two-dimensional crystals apparently have an infinite number of SP modes gathering around the surface plasma frequency $\omega_{\text {sp. }}$. The same figure seems to indicate that the number of resolved SP modes (RC modes) increases linearly (quadratically) with the number of grid points. In fact, existence of an infinite number of resonant modes can be inferred from the three-dimensional polaritonic photonic crystals ${ }^{28}$ and perfect corner reflectors. ${ }^{29}$ Likewise, for TM polarization, we have also observed the same behavior for MSP modes as well as RC modes. This point will be further discussed in the next section through the Rayleigh quotient of the eigensystem with the help of the interfacial variable.

Figures 7 and 8 show the band structures for a square array of square cylinders of negative index material with half-width $w / a=0.1$. For the negative index material with the effective permittivity (1) and permeability (2), a large number of frequency bands gathering around $\omega_{\text {sp }}$ occur for TE polarization. These resonant bands also occur in metallodielectric photonic crystals, ${ }^{30-32}$ which result from the strong photon-electron coupling. In the meanwhile, a large number of frequency bands occur also for TM polarization. The latter consist of branches of MSP modes gathering around the magnetic surface plasma frequency $\omega_{\mathrm{sm}}$, and the typical feature of resonant TM bands is similar to that of resonant TE bands. For TM polarization, there are no surface modes observed around the frequency $\omega_{\mathrm{sp}}$, while for TE polarization there are no surface modes observed around the 

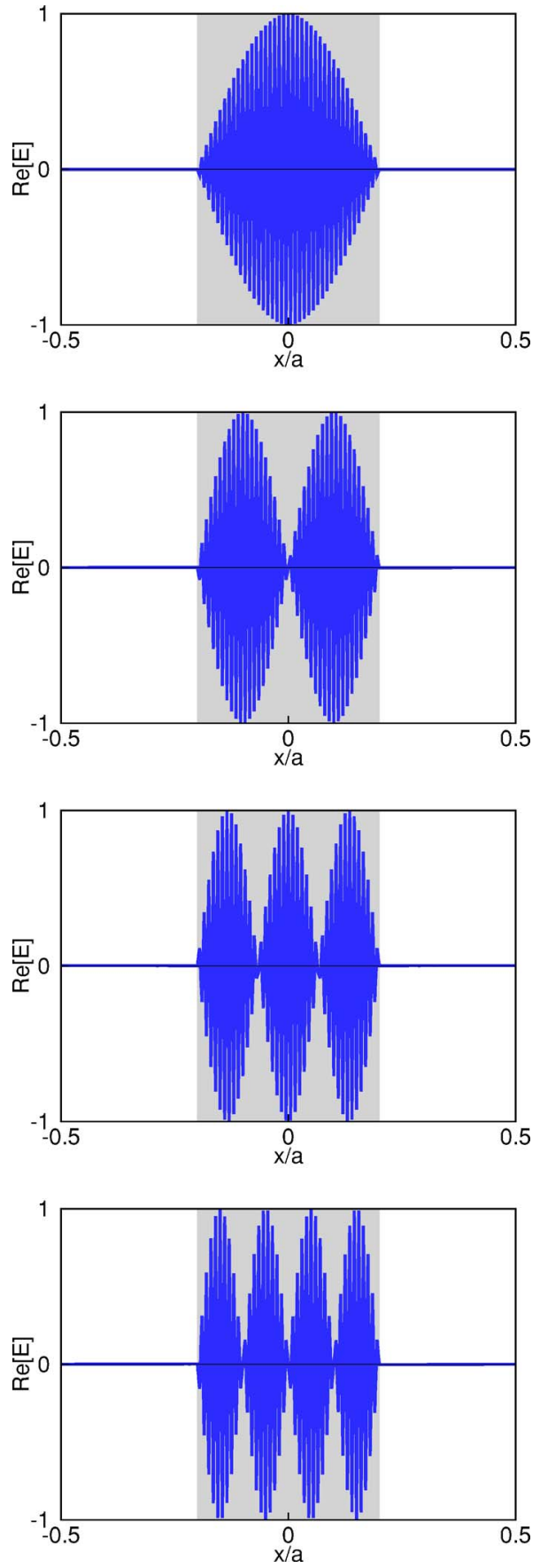

FIG. 5. (Color online) Four typical RC modes for TM polarization with $\omega a / 2 \pi c=0.4$ at the zone center $(k=0)$ with $\beta=0$ for a one-dimensional layered structure of negative index material of thickness $t / a=0.4$ where $\omega_{p} a / 2 \pi c=1.0, \omega_{0} a / 2 \pi c=0.4$, and $F$ $=0.56$.

frequency $\omega_{\mathrm{sm}}$. On the other hand, bulk modes are allowed in the frequency range with $\epsilon_{n}<0$ and $\mu_{n}<0$ because the equivalent permittivity or permeability $\epsilon_{n} \mu_{n}$ is positive. A similar periodic structure of two-dimensional checkerboard consisting of alternating positive and negative index materials was recently shown to possess peculiar imaging properties. $^{33}$

It is of interest to see what happens if the negative index material is made thin and connected. For this purpose, Figs.

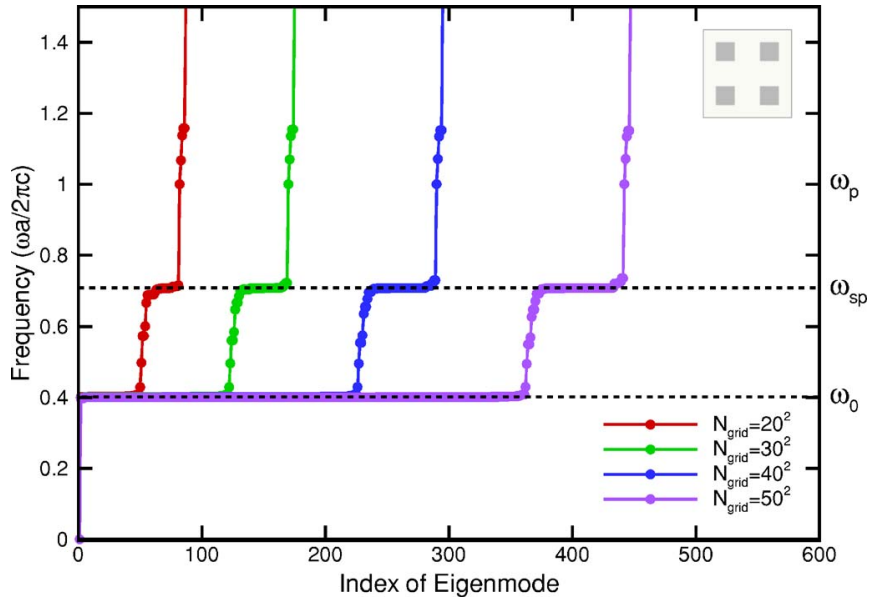

FIG. 6. (Color online) The eigenfrequencies for TE modes versus the index of eigenmode computed with different grid resolutions at the point $\Gamma$ for a square array of square cylinders of half width $w / a=0.2$ where $\omega_{p} a / 2 \pi c=1.0, \omega_{0} a / 2 \pi c=0.4$, and $F=0.56$.

9 and 10 show the band structures for a square array of grid cylinders with thickness $t / a=0.2$. Compared to Fig. 7 , the TM bands for thin grid structures spread more widely around $\omega_{\text {sm }}$ than for square cylinders. On the other hand, the TE bands for thin grid structures spread more widely around $\omega_{\mathrm{sp}}$ than for square cylinders. The band broadening corresponds to the degeneracy lifting of SP and MSP modes, which more easily appear in thin structures due to effective interaction of the modes on both sides of the structure. On the other hand,

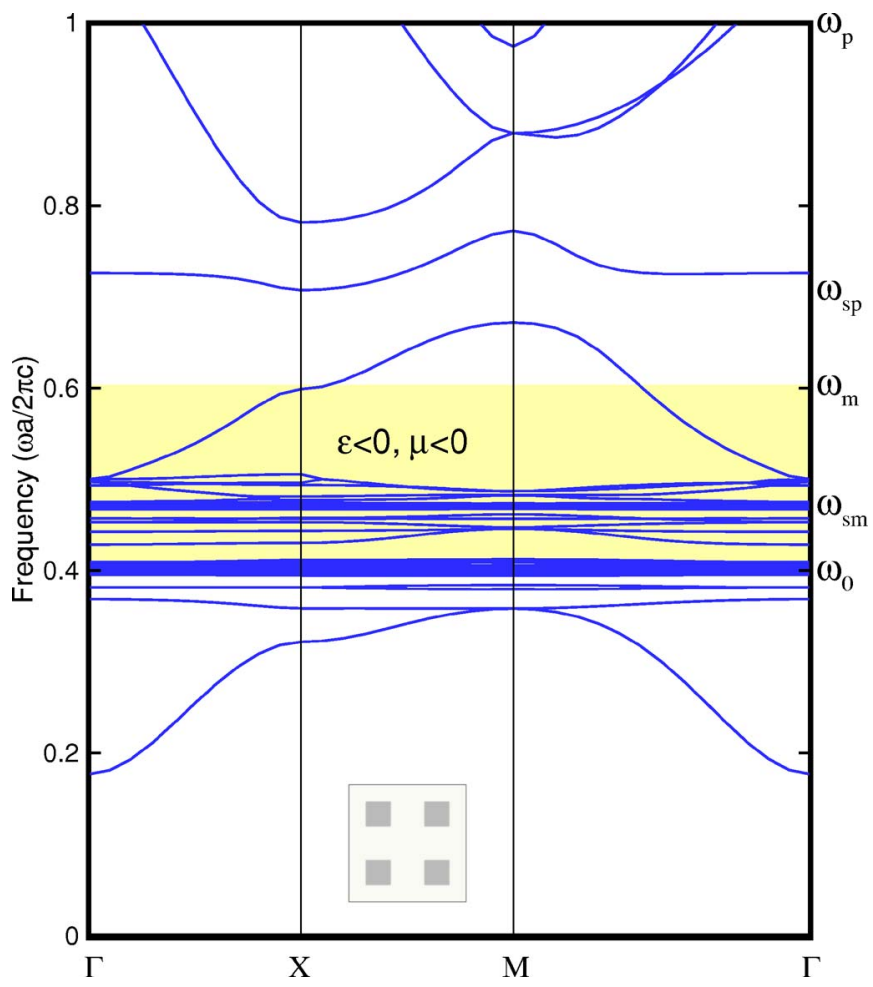

FIG. 7. (Color online) The TM band structures for a square array of square cylinders of negative index material with half-width $w / a=0.2$, where $\omega_{p} a / 2 \pi c=1.0, \omega_{0} a / 2 \pi c=0.4$, and $F=0.56$. 


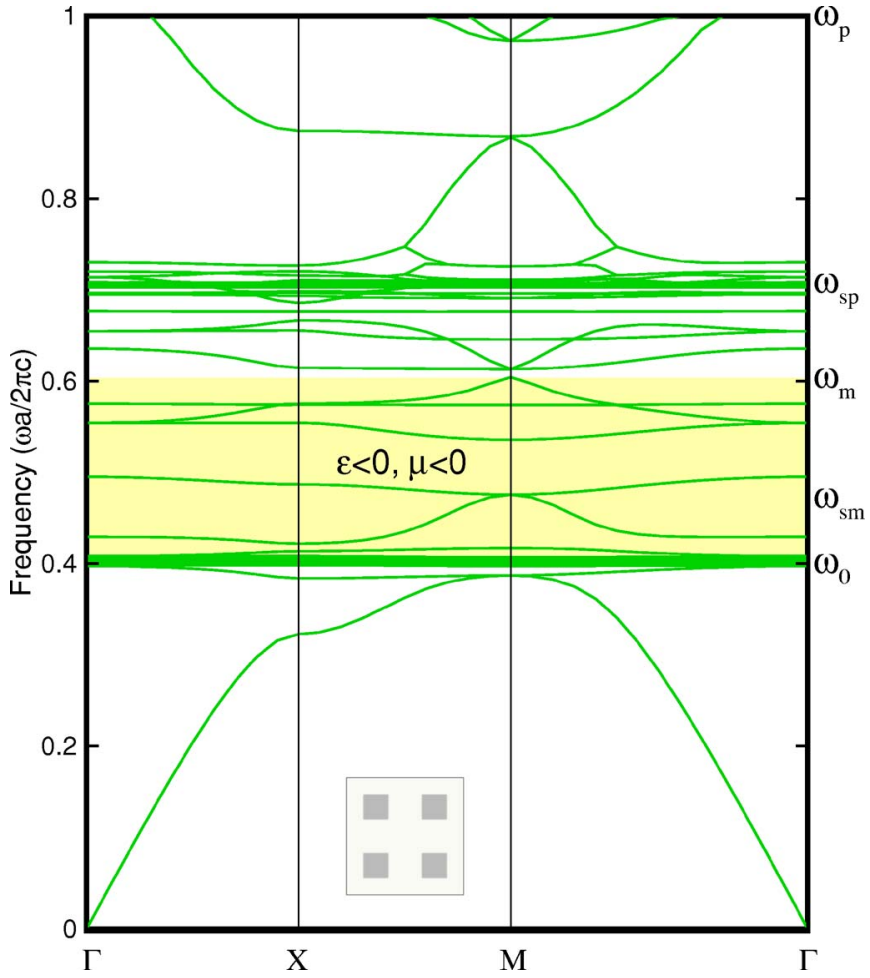

FIG. 8. (Color online) The TE band structures for a square array of square cylinders of negative index material with half-width $w / a=0.2$, where $\omega_{p} a / 2 \pi c=1.0, \omega_{0} a / 2 \pi c=0.4$, and $F=0.56$.

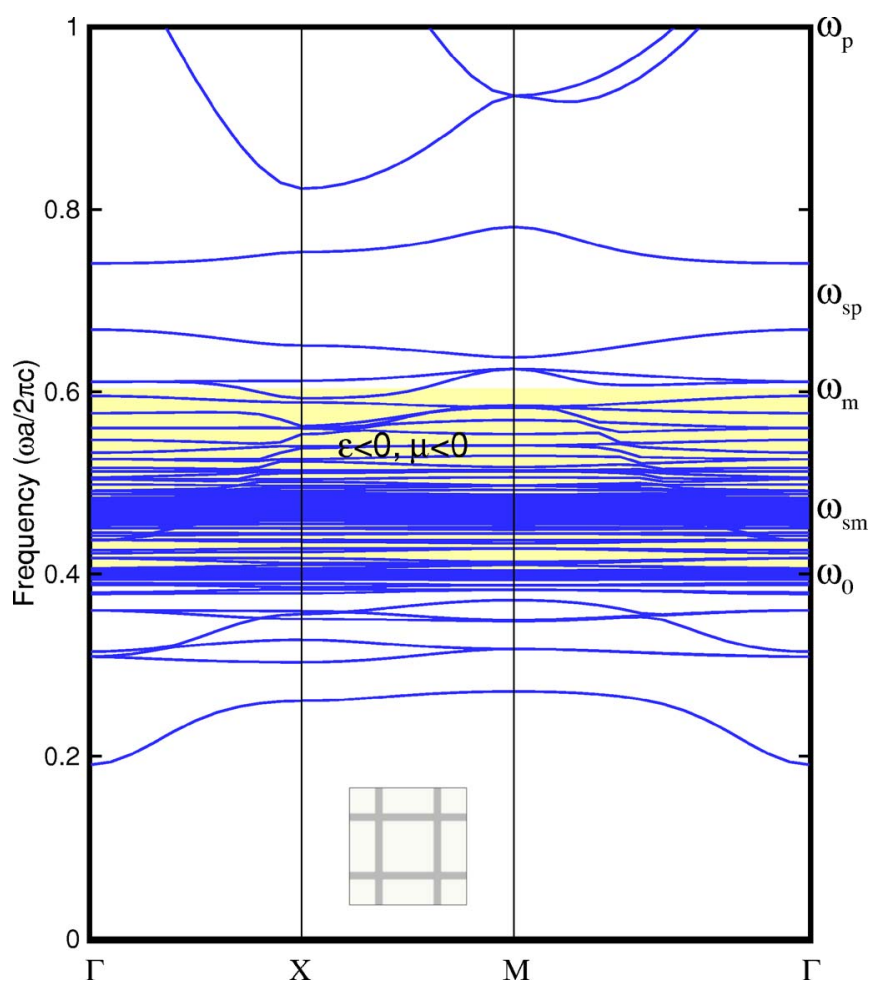

FIG. 9. (Color online) The TM band structures for a square array of grid cylinders of negative index material with the thickness ratio $t / a=0.1$, where $\omega_{p} a / 2 \pi c=1.0, \omega_{0} a / 2 \pi c=0.4$, and $F=0.56$.

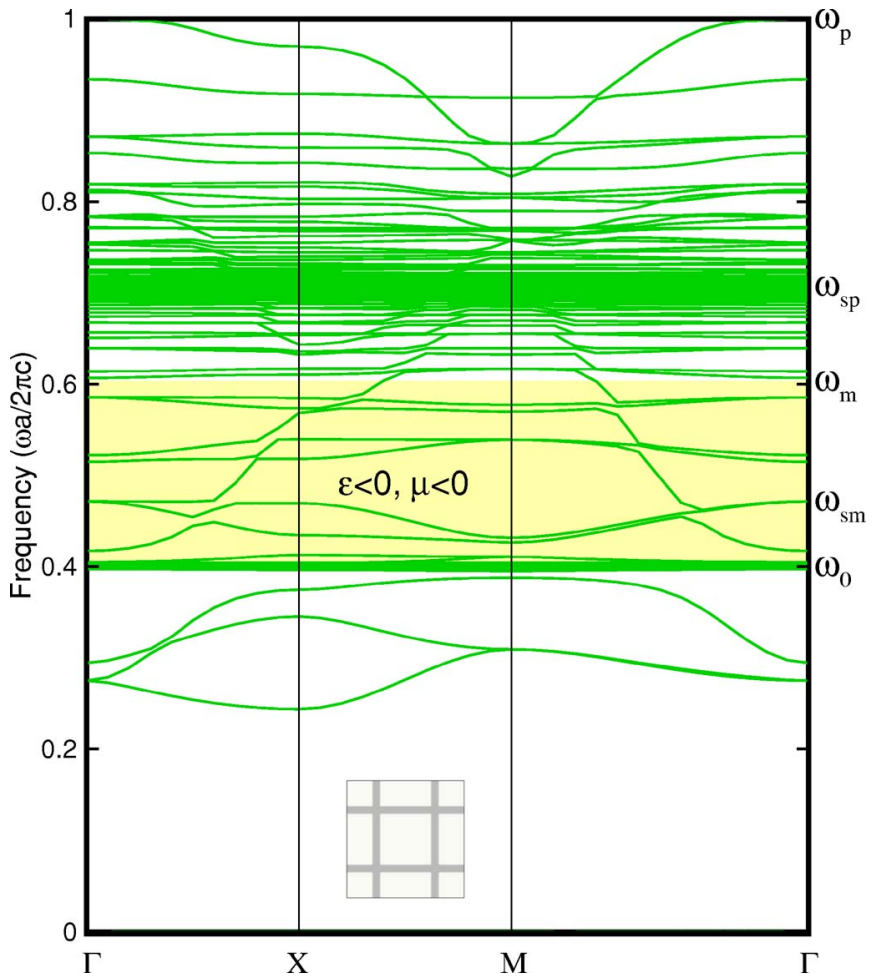

FIG. 10. (Color online) The TE band structures for a square array of grid cylinders of negative index material with the thickness ratio $t / a=0.1$, where $\omega_{p} a / 2 \pi c=1.0, \omega_{0} a / 2 \pi c=0.4$, and $F=0.56$.

connectivity of the structure allows existence of zerofrequency modes, in particular, for TE polarization for all wave vectors, and thus a zero-order band gap similar to the cutoff frequency in TM polarization is observed. These two points have also been discussed in Ref. 24 for photonic crystals of dispersive metals.

Figures 11 and 12 show four typical SP modes in magnitude for TE polarization and four typical MSP modes for TM polarization with frequencies near $\omega_{\mathrm{sp}}$ and $\omega_{\text {sm }}$, respectively. The fields are represented by a color level from blue to red indicating the magnitude from zero to the maximum unity, and the black line denotes the boundary of the negative index material. A very sharp feature of the field pattern near the interface is observed for SP modes as well as MSP modes. Different variations of the fields along the interface account for the high degeneracy around $\omega_{\mathrm{sp}}$ or $\omega_{\mathrm{sm}}$, and an infinite number of SP or MSP modes would be expected.

Resonant cavity modes. Next, we consider the RC modes with frequencies lying immediately above the resonant frequency $\omega_{0}$. In Figs. 7-10, even more flattened bands for both TE and TM modes gathering around $\omega_{0}$ from above are observed. This is analogous to the one-dimensional case, and these modes have similar properties of RC modes that appear in two-dimensional polaritonic crystals. ${ }^{20}$ Figure 13 shows four typical RC modes in magnitude for TE polarization with frequencies near $\omega_{0}$ at the point $\Gamma$ for a square array of square cylinders of half-width $w / a=0.3$.

These bulk modes correspond to very large dielectric constants where $\varepsilon_{n} \mu_{n} \rightarrow \infty$ as the frequency approaches $\omega_{0}$ from above. This is consistent with the resonances of the square 

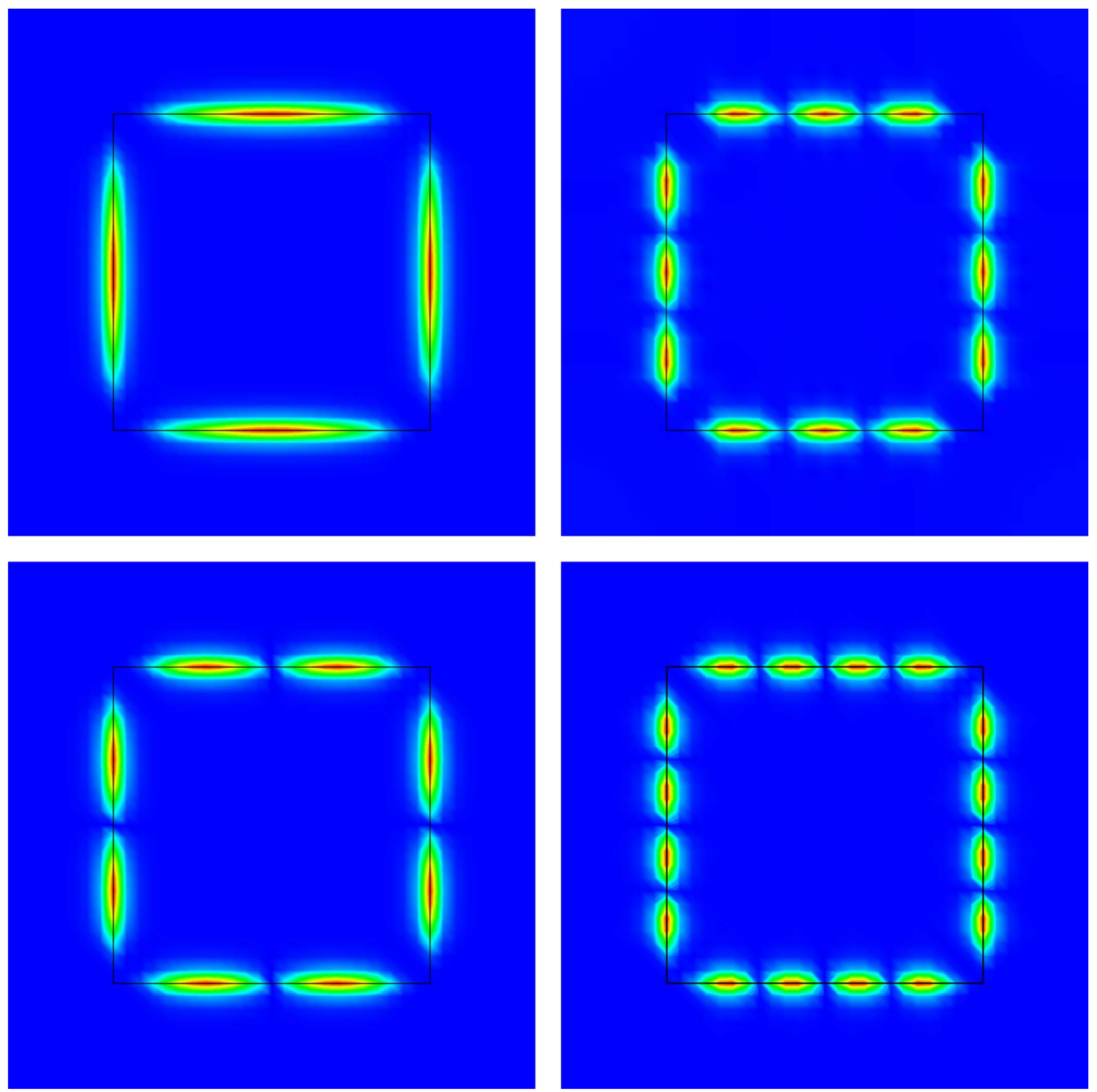

FIG. 11. (Color online) Four typical SP modes in magnitude for TE polarization with frequencies near $\omega_{\mathrm{sp}}$ at the point $\Gamma$ for a square array of square cylinders of negative index material of half-width $w / a=0.3$ where $\omega_{p} a / 2 \pi c=1.0, \omega_{0} a / 2 \pi c=0.4$, and $F=0.56$.

cavity or the metallic waveguide modes with frequencies $\omega_{k l}$ determined by two free indices $k$ and $l^{34}$

$$
\omega_{k l}=\frac{\pi c}{2 w \sqrt{\varepsilon_{k l} \mu_{k l}}} \sqrt{k^{2}+l^{2}},
$$

where $\varepsilon_{k l}=1-\omega_{p}^{2} / \omega_{k l}^{2}$ and $\mu_{k l}=(1-F)\left(\omega_{k l}^{2}-\omega_{m}^{2}\right)\left(\omega_{k l}^{2}-\omega_{0}^{2}\right)$. Solving Eq. (28) for $\omega_{k l}$ yields

$$
\omega_{k l}^{2}=\frac{2 \beta_{k l}}{\alpha_{k l}+\sqrt{\alpha_{k l}^{2}-4 \beta_{k l}}},
$$

where $\quad \alpha_{k l}=\omega_{p}^{2}+\omega_{m}^{2}+\Omega_{k l}^{2}, \quad \beta_{k l}=\Omega_{k l}^{2} \omega_{0}^{2}+\omega_{p}^{2} \omega_{m}^{2}, \quad$ and $\quad \Omega_{k l}$ $=\pi c \sqrt{k^{2}+l^{2}} 2 w \sqrt{1-F}$ with $w$ the half-width of the square cavity. The expression (29) indicates that $\omega_{0}$ is the lower limit frequency of RC modes for if $\Omega_{k l}$ goes to infinity, $\omega_{k l}$ approaches $\omega_{0}$. On the other hand, if $\Omega_{k l}$ goes to $0, \omega_{k l}$ approaches $\left[\omega_{m}^{2}-\Omega_{k l}^{2}\left(\omega_{m}^{2}-\omega_{0}^{2}\right) /\left(\omega_{p}^{2}-\omega_{m}^{2}\right)\right]^{1 / 2}$. Therefore, for a 

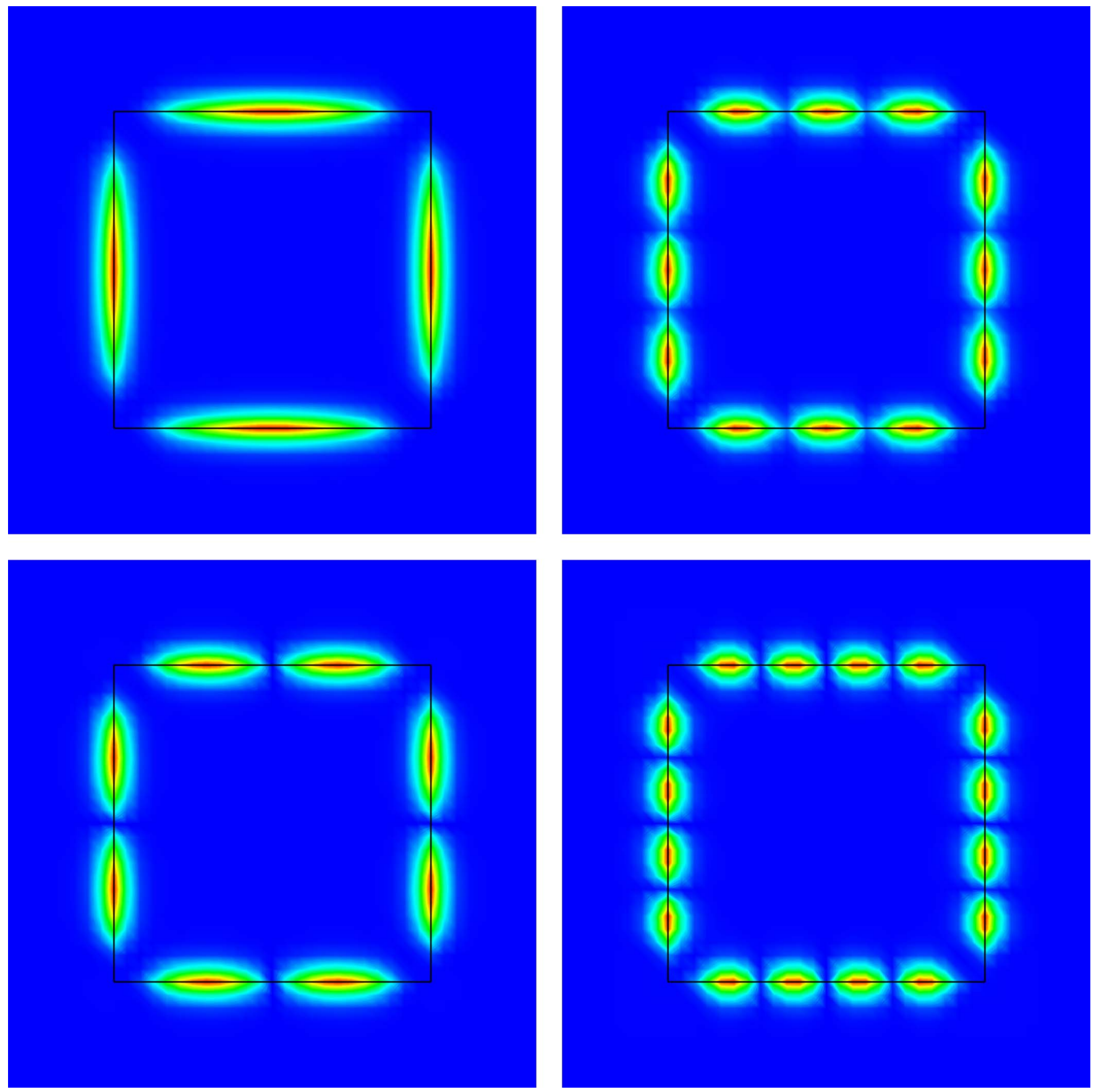

FIG. 12. (Color online) Four typical MSP modes in magnitude for TM polarization with frequencies near $\omega_{\mathrm{sp}}$ at the point $\Gamma$ for a square array of square cylinders of negative index material of half-width $w / a=0.3$ where $\omega_{p} a / 2 \pi c=1.0, \omega_{0} a / 2 \pi c=0.4$, and $F=0.56$.

larger fraction of the negative index material, that is, large value of $w / a$, the resonant bands spread more widely.

Asymmetric surface modes. As in the case of the onedimensional structures, at the interface the asymmetric surface (AS) mode decays 5.25 times faster inside the negative index material than outside [cf. Eq. (25)]. The present grid resolution shows that the frequency range of AS modes is highly concentrated on a very narrow region lying immediately below the resonance frequency $\omega_{0}$ where the product $\epsilon_{n} \mu_{n}$ is largely negative. It is therefore expected that the material with negative $\epsilon_{n} \mu_{n}$ effectively expells the fields, presenting itself like a mask region. Finer grid resolutions show that the number of AS modes increases with increasing the number of grid points. This is in contrast to only two such modes in one-dimensional structures, and there might be an infinite number of AS modes in two dimensions. The field pattern of AS modes with different variations along the interface may be responsible for possible infinite degeneracy. Comparison between Figs. 8 and 10 shows that the thinner negative index material allows effective broadening of SP and MSP modes, but not AS modes. This is because the more rapidly decaying rate of the AS modes in the negative index material does not allow overlap of the field on opposite sides of the material, and therefore has no effect in lifting their 

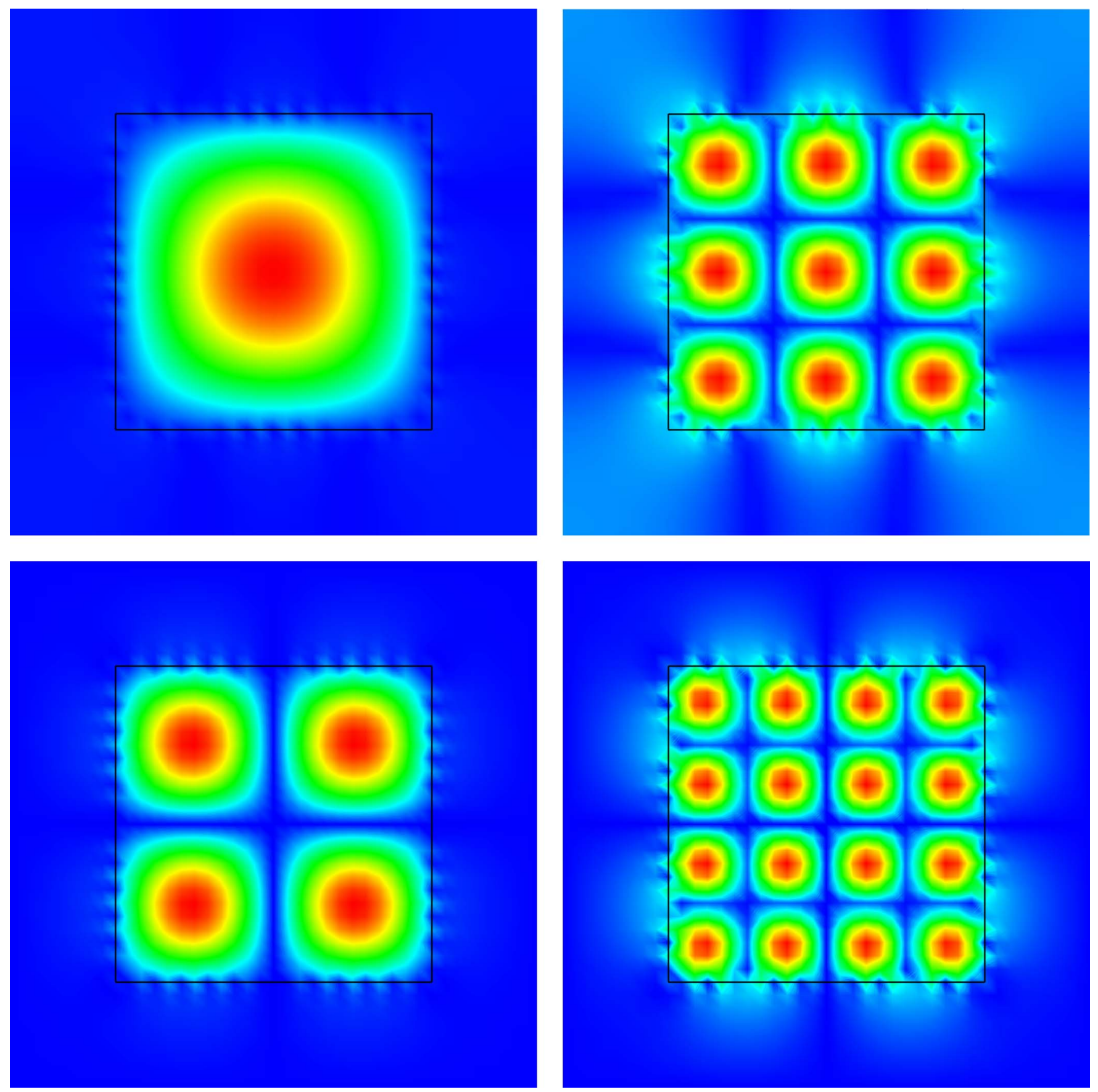

FIG. 13. (Color online) Four typical RC modes in magnitude for TM polarization with frequencies near $\omega_{0}$ at the point $\Gamma$ for a square array of square cylinders of negative index material of half-width $w / a=0.3$ where $\omega_{p} a / 2 \pi c=1.0, \omega_{0} a / 2 \pi c=0.4$, and $F=0.56$.

degeneracy. It must also be noted that in the surrounding dielectric medium, the field of an AS mode in magnitude does diminish monotonically (rather than exhibit an oscillatory pattern) as the distance from the interface is increased. The four AS modes shown in Fig. 14 have different degrees of oscillation along the interface, and in general the mode with a higher degree decays more rapidly away from the interface than that with a lower degree.

The frequency ranges of the various modes described above are conveniently summarized in Table I.

\section{Interfacial variable}

In the present approach, the interfacial variables $S_{E}$ in Eq. (21) and $S_{H}$ in Eq. (22) are introduced to be the weighted differences of the normal derivatives of the $E$ and $H$ fields, respectively, on two sides of the interface. These variables serve as a measure of the local strengths of SP or MSP modes at the interface, and their meanings can be further manifest through the formulations of the Rayleigh quotients for the eigensystems (5) and (6). It is known that the eigenfrequency corresponds to minimization of the Rayleigh quo- 

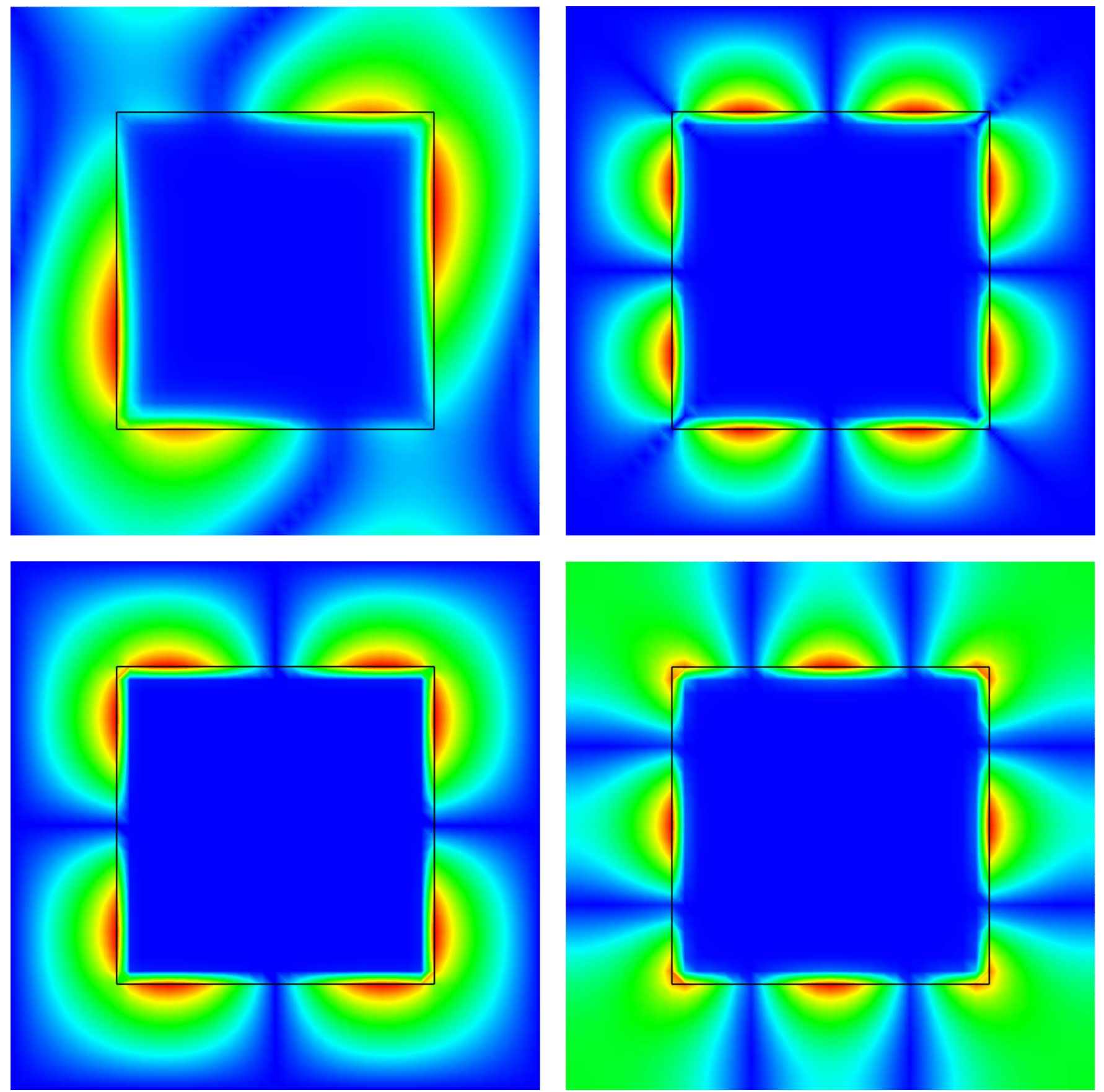

FIG. 14. (Color online) Four typical AS modes in magnitude for TE polarization with frequencies near $\omega_{0}$ at the point $\Gamma$ for a square array of square cylinders of negative index material of half-width $w / a=0.3$ where $\omega_{p} a / 2 \pi c=1.0, \omega_{0} a / 2 \pi c=0.4$, and $F=0.56$.

tient under the constraint that the corresponding eigenfunction be orthogonal to all previously obtained eigenfunctions. The Rayleigh quotient for a standard eigensystem $\mathcal{L} \phi=\Lambda \phi$, is given by

$$
R_{\phi}=\frac{\langle\phi, \mathcal{L} \phi\rangle}{\langle\phi, \phi\rangle},
$$

where $\langle f, g\rangle=\int_{V_{\text {cell }}} f^{*} g d \tau$ denotes the inner product of $f$ and $g$ over the unit cell $V_{\text {cell }}$. For a linear operator $\mathcal{L}$, Eq. (30) can be used directly, while for a nonlinear operator, the Rayleigh
TABLE I. Various modes for TM and TE polarizations for different optical properties.

\begin{tabular}{ccc}
\hline \hline Optical constants & TM polarization & TE polarization \\
\hline$\omega \approx \omega_{\mathrm{sp}}$ & & SP modes \\
$\omega \approx \omega_{\mathrm{sm}}$ & MSP modes & \\
$\varepsilon<0, \mu \approx-\infty$ & RC modes & RC modes \\
$\varepsilon<0, \mu \approx+\infty$ & & AS modes \\
$\varepsilon<0, \mu<0$ & Bulk modes & Bulk modes \\
\hline \hline
\end{tabular}


quotient $R_{\phi}$ must be obtained in a slightly different manner. Based on the interfacial operator approach developed in the preceding section, we rewrite the eigensystems (12) and (16) for TE modes in the dielectric and the negative index material, respectively, as

$$
\begin{gathered}
\Lambda^{2}\left(\mu_{d} H\right)+\Lambda\left(\frac{1}{\varepsilon_{d}} \nabla^{2} H\right)=0, \\
\Lambda^{2}\left(\mu_{\infty} H\right)-\Lambda\left[\mu_{\infty}\left(\Lambda_{p}+\Lambda_{m}\right) H-\nabla^{2} H\right]+\mu_{\infty} \Lambda_{p} \Lambda_{m} H-\Lambda_{0} \nabla^{2} H \\
\quad=0 .
\end{gathered}
$$

Taking inner product of $H$ with each term of the above two equations, adding them together, performing the integration by parts and using the Bloch condition (8), we obtain a quadratic expression of the Rayleigh quotient $R_{H}$,

$$
R_{H}^{2} A-R_{H} B+C=0,
$$

or, equivalently,

$$
R_{H}=\frac{B \pm \sqrt{B^{2}-4 A C}}{2 A}
$$

where

$$
\begin{gathered}
A=\mu_{d} \int_{V_{d}}|H|^{2} d \tau+\mu_{\infty} \int_{V_{n}}|H|^{2} d \tau, \\
B=\int_{S_{n}} H^{*} S_{H} d a+\frac{1}{\varepsilon_{d}} \int_{V_{d}}|\nabla H|^{2} d \tau+\int_{V_{n}}\left[|\nabla H|^{2}\right. \\
\left.+\mu_{\infty}\left(\Lambda_{p}+\Lambda_{m}\right)|H|^{2}\right] d \tau, \\
C=-\left.\Lambda_{0} \int_{S_{n}} H^{*} \frac{\partial H}{\partial n}\right|_{-} d a+\Lambda_{0} \int_{V_{n}}|\nabla H|^{2} d \tau \\
+\mu_{\infty} \Lambda_{p} \Lambda_{m} \int_{V_{n}}|H|^{2} d \tau,
\end{gathered}
$$

with $V_{d}$ and $V_{n}$ denoting the volumes of the dielectric and the negative index material, respectively, of the unit cell, and $S_{n}$ denoting the surface of $V_{n}$. In the expression of $B$, the interfacial variable $\left.S_{H} \equiv \frac{1}{\varepsilon_{d}} \frac{\partial H}{\partial n}\right|_{+}-\left.\frac{\partial H}{\partial n}\right|_{-}$[cf. Eq. (22)] appears in the surface integral term, which accounts for the contribution of the strength of SP mode to the eigenfrequency. Note also that only the normal derivative of the $H$ field $(\partial H / \partial n)$ occurs in the surface integral, and therefore tangential variation of the $H$ field will not change the value of $R_{H}$ as well as the eigenfrequency. This can be used to explain the highly degenerate nature of SP modes, that is, a large number of different patterns of SP modes possessing an identical oscillation frequency (see also Refs. 21 and 24).

Likewise, the eigensystems (11) and (15) for TM modes in the dielectric and the negative index material, respectively, are rewritten as

$$
\Lambda^{2}\left(\varepsilon_{d} E\right)+\Lambda\left(\frac{1}{\mu_{d}} \nabla^{2} E\right)=0
$$

$$
\Lambda^{2} E-\Lambda\left(\left(\Lambda_{p}+\Lambda_{m}\right) E-\frac{1}{\mu_{\infty}} \nabla^{2} E\right)+\Lambda_{p} \Lambda_{m} E-\frac{\Lambda_{0}}{\mu_{\infty}} \nabla^{2} E=0 .
$$

Using the same technique, a quadratic expression of the Rayleigh quotient $R_{E}$ for TM modes is given as

$$
R_{E}^{2} D-R_{E} F+G=0
$$

where

$$
\begin{gathered}
D=\varepsilon_{d} \int_{V_{d}}|E|^{2} d \tau+\int_{V_{n}}|E|^{2} d \tau, \\
F=\int_{S_{n}} E^{*} S_{E} d a+\frac{1}{\mu_{d}} \int_{V_{d}}|\nabla E|^{2} d \tau \\
+\int_{V_{n}}\left(\frac{1}{\mu_{\infty}}|\nabla E|^{2}+\left(\Lambda_{p}+\Lambda_{m}\right)|E|^{2}\right) d \tau, \\
G=-\left.\frac{\Lambda_{0}}{\mu_{\infty}} \int_{S_{n}} E^{*} \frac{\partial E}{\partial n}\right|_{-} d a+\frac{\Lambda_{0}}{\mu_{\infty}} \int_{V_{n}}|\nabla E|^{2} d \tau \\
+\Lambda_{p} \Lambda_{m} \int_{V_{n}}|E|^{2} d \tau .
\end{gathered}
$$

In the expression of $F$, the interfacial variable $\left.S_{E} \equiv \frac{1}{\mu_{d}} \frac{\partial E}{\partial n}\right|_{+}$ $-\left.\frac{1}{\mu_{\infty}} \frac{\partial E}{\partial n}\right|_{-}$[cf. Eq. (21)] appears in the surface integral term, which accounts for the contribution of the strength of MSP mode to the eigenfrequency.

A close inspection of the derivations of Eqs. (33) and (37) reveals that all the three terms in $C$ or $G$ are contributed exclusively by the negative index material. The singly most significant difference between the two Rayleigh quotients is the appearance of $S_{H}$ in $B$ for TE modes, and $S_{E}$ in $F$ for TM modes. The close resemblance between $S_{E}$ and $S_{H}$ in the expressions of the Rayleigh quotients also explains the existence of a magnetic analog of SP modes for TM polarization. In this regard, the interfacial variable $S_{H}$ or $S_{E}$ is not only introduced to be an auxiliary variable for solving the eigenfrequency, but also serves as a quantitative measure to the strength of SP or MSP mode. It is also noted that in the expression of the Rayleigh quotients $R_{H}$ and $R_{E}$, only normal derivatives of the fields are incorporated in the integrals, and variation of the fields along the interface will not change the value of the Rayleigh quotient well as the eigenfrequency. Therefore, the interface can sustain, in principle, an infinite number of degenerate modes.

\section{CONCLUDING REMARKS}

In conclusion, we have computed the band structures and various surface modes for periodic structures made of negative index materials by the interfacial operator approach. It was shown that all the differences between TE and TM modes consist in the different interface conditions which are employed to formulate the interfacial operator approach. The crucial step is the introduction of the interfacial variable at 
the interface between the negative index material and the surrounding dielectric medium. The interfacial variable leads to a finite difference formulation for transforming the apparently nonlinear eigenvalue problem for the eigenmodes and eigenfrequencies to a quadratic eigenvalue problem, which is further reduced to a linear eigensystem.

In different ranges of frequencies, we have identified five types of modes: (a) SP modes distributed around $\omega_{\mathrm{sp}}$, (b) MSP modes distributed around $\omega_{\mathrm{sm}}$, (c) trapped modes or RC modes lying immediately above $\omega_{0}$, (d) AS modes lying immediately below $\omega_{0}$, and (e) other bulk modes in the range of negative material properties. The SP and MSP modes, respectively, are pertaining to individual negative electric permittivity and negative magnetic permeability. In contrast, the RC modes and the AS modes are closely associated with the large positive product $\epsilon_{n} \mu_{n}$ and large negative product $\epsilon_{n} \mu_{n}$ near the resonance frequency $\omega_{0}$. The present grid resolution indicates that there is possibly an infinite number of SP,
MSP, and AS modes in two dimensions. The distinctions between SP, MSP, and AS modes were examined by the interface conditions and the Rayleigh quotients, which also help explain the infinite degeneracy of these surface modes. It was also shown that the thinner negative index material allows effective broadening of SP and MSP modes, but not AS modes. This is because the more rapidly decaying rate of the AS modes in the negative index material does not allow overlap of the field on opposite sides of the material, and therefore has no effect in lifting their degeneracy.

\section{ACKNOWLEDGMENTS}

This work was supported in part by National Science Council of the Republic of China under Contracts Nos. NSC 94-2212-E-002-047 and NSC 94-2212-E-002-076, and the Ministry of Economic Affairs of the Republic of China under Contract No. MOEA 95-EC-17-A-08-S1-0006.
*Electronic address: chern@iam.ntu.edu.tw

$\dagger$ Electronic address: mechang@gate.sinica.edu.tw

${ }^{1}$ V. G. Veselago, Sov. Phys. Usp. 10, 509 (1968).

${ }^{2}$ J. B. Pendry, A. J. Holden, D. J. Robbins, and W. J. Stewart, J. Phys.: Condens. Matter 10, 4785 (1998).

${ }^{3}$ J. B. Pendry, A. J. Holden, D. J. Robbins, and W. J. Stewart, IEEE Trans. Microwave Theory Tech. 47, 2075 (1999).

${ }^{4}$ D. R. Smith and N. Kroll, Phys. Rev. Lett. 85, 2933 (2000).

${ }^{5}$ S. Linden, C. Enkrich, M. Wegener, J. Zhou, T. Koschny, and C. M. Soukoulis, Science 306, 1351 (2004).

${ }^{6}$ L. D. Landau, E. M. Lifshitz, and L. P. Pitaevskii, Electrodynamics of Continuous Media, 2nd ed. (Butterworth-Heinenann, Oxford, 1984).

${ }^{7}$ J. B. Pendry and S. O’Brien, J. Phys.: Condens. Matter 14, 7409 (2002).

${ }^{8}$ A. Hartstein, E. Burstein, A. A. Maradudin, R. Brewer, and R. F. Wallis, J. Phys. C 6, 1266 (1973).

${ }^{9}$ R. E. Camley and D. L. Mills, Phys. Rev. B 26, 1280 (1982).

${ }^{10}$ M. R. F. Jensen, S. A. Feiven, T. J. Parker, and R. E. Camley, Phys. Rev. B 55, 2745 (1997).

${ }^{11}$ O. Toader and S. John, Phys. Rev. E 70, 046605 (2004).

${ }^{12}$ V. Kuzmiak, A. A. Maradudin, and A. R. McGurn, Phys. Rev. B 55, 4298 (1997).

${ }^{13}$ M. M. Sigalas, C. M. Soukoulis, C. T. Chan, and K. M. Ho, Phys. Rev. B 49, 11080 (1994).

${ }^{14}$ A. Moroz, J. Phys.: Condens. Matter 6, 171 (1994).

${ }^{15}$ A. Moroz, Phys. Rev. B 51, 2068 (1995).

${ }^{16}$ A. Moroz and A. Tip, Phys. Lett. A 235, 195 (1997).

${ }^{17}$ A. Modinos, N. Stefanou, and V. L. Yannopapas, Opt. Express 8, 197 (2001).
${ }^{18}$ T. Ito and K. Sakoda, Phys. Rev. B 64, 045117 (2001).

${ }^{19}$ E. Moreno, D. Erni, and C. Hafner, Phys. Rev. B 65, 155120 (2002).

${ }^{20}$ K. C. Huang, P. Bienstman, J. D. Joannopoulos, K. A. Nelson, and S. Fan, Phys. Rev. Lett. 90, 196402 (2003).

${ }^{21}$ C. C. Chang, R. L. Chern, C. C. Chang, and R. R. Hwang, Phys. Rev. B 72, 205112 (2005).

${ }^{22}$ R. L. Chern, C. C. Chang, and C. C. Chang, Phys. Rev. B 73, 235123 (2006).

${ }^{23}$ H. Raether, Surface Plasmons on Smooth and Rough Surfaces and on Gratings (Springer-Verlag, Berlin, 1988).

${ }^{24}$ R. L. Chern, C. C. Chang, and C. C. Chang, Phys. Rev. E 73, 036605 (2006).

${ }^{25}$ R. Ruppin, Phys. Lett. A 277, 61 (2000).

${ }^{26}$ R. Ruppin, J. Phys.: Condens. Matter 13, 1811 (2001).

${ }^{27}$ S. Darmanyan, M. Neviére, and A. Zakhidov, Opt. Commun. 225, 233 (2003).

${ }^{28}$ G. Gantzounis and N. Stefanou, Phys. Rev. B 72, 075107 (2005).

${ }^{29}$ S. Guenneau, B. Gralak, and J. Pendry, Opt. Lett. 30, 1204 (2005).

${ }^{30}$ A. Moroz, Phys. Rev. Lett. 83, 5274 (1999).

${ }^{31}$ H. van der Lem and A. Moroz, J. Opt. A, Pure Appl. Opt. 2, 395 (2000).

${ }^{32}$ H. van der Lem, A. Tip, and A. Moroz, J. Opt. Soc. Am. B 20, 1334 (2003).

${ }^{33}$ S. Guenneau, A. Vutha, and S. Ramakrishna, New J. Phys. 7, 164 (2005).

${ }^{34}$ K. C. Huang, P. Bienstman, J. D. Joannopoulos, K. A. Nelson, and S. Fan, Phys. Rev. B 68, 075209 (2003). 